\title{
Chemical and physical properties of interstellar dust
}

\author{
A. G. G. M. Tielens \\ Leiden Observatory, Leiden University, PO Box 9513, NL-2300 RA Leiden, the Netherlands \\ email: tielens@strw.leidenuniv.nl
}

\begin{abstract}
The characteristics of interstellar dust reflect a complex interplay between stellar injection of stardust, destruction in the ISM, and regrowth in clouds. Astronomical observations and analysis of stardust isolated from meteorites have revealed a highly diverse interstellar and circumstellar grain inventory, including both amorphous materials and highly crystalline compounds (silicates and carbon). This review summarizes this dust budget and inventory. Interstellar dust is highly processed during its sojourn from its birthsite (stellar ejecta) to its incorporation into protoplanetary systems. Processing by strong shocks due to supernova explosions is particularly important. Sputtering by impacting gas ions in shocks in the intercloud medium of the ISM is counteracted by accretion in cloud phases and their balance sets the observed, interstellar, elemental depletion patterns. Astronomical and meteoritical-stardust evidence for these processes is reviewed and it is concluded that dust formation in the ISM is very rapid. Not surprisingly, the characteristics of interstellar dust are expected to vary widely reflecting local stellar sources, the effects of SNe processing, and the interstellar accretion process.
\end{abstract}

\section{Introduction}

The presence of small dust grains in the interstellar medium of galaxies near and far is very apparent through extinction of stellar and nebular photons, through scattered light, through optical and infrared polarization, and through infrared emission. Polycyclic Aromatic Hydrocarbon (PAHs) molecules, the extension of the interstellar grain size distribution into the molecular domain, are equally prominent in images and spectra of galaxies at mid-IR wavelengths. In addition, many of the key processes that drive the evolution of galaxies - including star and planet formation as well as the accretion onto central black holes - occur deeply inside dust enshrouded regions. Hence, understanding the characteristics of interstellar dust is of key importance for analyzing observations of galaxies and their evolutionary processes: star formation and black hole interaction.

In addition, small dust grains play an important role in the physical and chemical evolution of the interstellar medium of galaxies. Dust grains shield the gas from the destructive effects of FUV photons. In addition, dust grains provide surfaces for the formation of $\mathrm{H}_{2}$ and other molecules. Such species play a key role in the star formation process because they control the degree of ionization and therefore the coupling of the gas to magnetic fields. Moreover, molecules dominate the cooling of dense clouds, facilitating gravity to overcome thermal supporting forces. Small grains and PAHs also dominate the heating of neutral gas through the photo-electric effect and thereby the physical conditions and the phase structure of the ISM. Last but not least, dust grains are the building blocks of cometesimals and planetesimals in the first steps towards the formation of planetary systems.

Studies of interstellar dust are therefore of key importance for our understanding of the evolution of galaxies. The recent observations that relatively large amounts of dust are present in galaxies as early as redshifts of 6 (Bertoldi et al. 2003) drive a number of key 


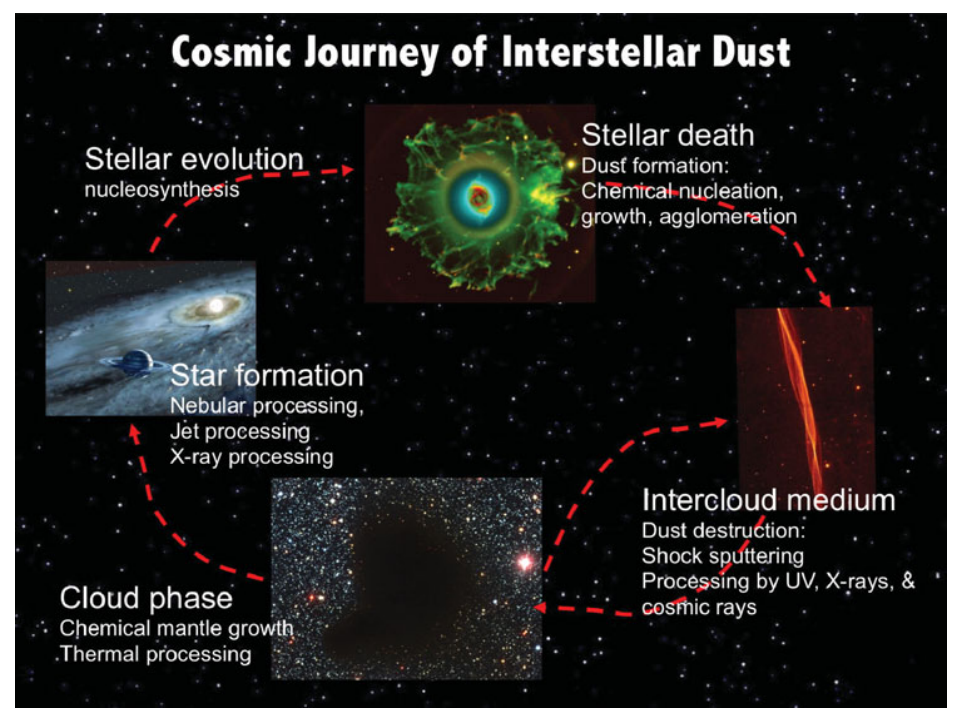

Figure 1. The lifecycle of large molecules and grains in space. At the end of their life, stars return most of their material back to the interstellar medium enriched by their nucleosynthetic products. In the interstellar medium, the ejected material mixes with material left over from the big bang and with material from other stars. Eventually, new stars and planetary systems are born from this material. During this cosmic cycle, materials are heavily processed. Some of these processes are indicated in this figure. (Figure adapted from Tielens 2005).

questions: "What are the sources of dust and how did their contribution vary over the age of the Universe ?", "What processes modify the dust characteristics in the interstellar medium ?", and "How did this dust influence the evolution of galaxies ?". In this review, I will address a number of these issues and indicate future observational opportunities that may help us address the characteristics and role of dust in the Universe.

\section{The lifecycle of interstellar dust}

The lifecycle of dust in space starts with the nucleation and growth of high temperature condensates such as silicates, oxides, graphite, and carbides at high densities and temperatures in the ejecta from stars (Fig. 1). This ejected material is rapidly mixed with other gas and dust in the interstellar medium of galaxies. In the interstellar medium, dust cycles many times between the intercloud and cloud phases on a very fast timescale $\left(\simeq 3 \times 10^{7} \mathrm{yr}\right)$. In the low density, warm neutral and ionized intercloud media, material is processed by strong shocks driven by supernova explosions. The hot gasses in the shock sputter atoms from the dust while high velocity collisions among grains lead to vaporization, melting, phase transformation, and shattering of the projectile and target. In the denser media - diffuse and dense clouds -, gas phase species accrete onto grains forming a mantle. Coagulation may also play a role in increasing the grain size inside clouds. If the grain survives the onslaught of interstellar shocks, eventually, during one of these cycles, a grain may find itself in a dense cloud core when this core becomes gravitationally unstable against collapse. The grain may then wind up in the star or in the surrounding planet-forming disk and eventually become part of a planet-forming body. The complete cycle from injection by a star until formation of a new star and any associated planets typically takes some $3 \times 10^{9} \mathrm{yr}$. 


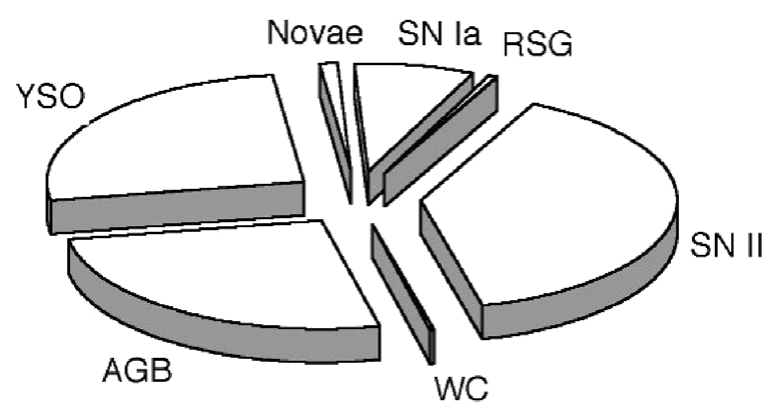

Figure 2. The contribution of different stellar sources to the interstellar dust budget. For details, see $\S 3$. Note that all these values are very uncertain. (Taken from Tielens 2001).

\section{Sources of stardust}

Stars in the last stages of their evolution return much of their material to the ISM. For most stars, as these ejecta expand and cool, small dust grains nucleate and grow when the temperature drops to about $1500-1000 \mathrm{~K}$, depending on the material and the chemical kinetics (Cherchneff 2000, Cherchneff \& Dwek 2010). The gas mass return rate to the ISM - mostly $\mathrm{H}$ and $\mathrm{He}$ - is dominated by Asymptotic Giant Branch stars (AGB), as expected since these objects are the descendants of low mass stars that dominate the stellar mass budget and the Milky Way is approximately in steady state. In terms of condensible elements, the mass return rate is about equally due to AGB stars and SNe (Tielens 2005). Observations show that AGB stars are important contributors to the stardust budget of the Milky Way, returning some $8 \mathrm{M}_{\odot} \mathrm{kpc}^{-2} \mathrm{Myr}^{-1}$ in solid form. By comparison, red supergiants, the descendants of massive stars, return only a paltry $0.2 \mathrm{M}_{\odot} \mathrm{kpc}^{-2} \mathrm{Myr}^{-1}$ of dust. The contribution of SNe to the dust mass budget is unclear. If all the condensible material were turned into dust, type II SNe might contribute some $12 \mathrm{M}_{\odot} \mathrm{kpc}^{-2} \mathrm{Myr}^{-1}$. Observationally, dust is known to form typically between 300 and 1000 days after the SN explosion as revealed by a sudden drop in optical light, a concommittant increase in IR emission, and the development of a pronounced bluered asymmetry in the emission line profiles of the ejecta (Wooden et al. 1993, Lucy et al. 1989). However, IR studies of core collapse SNe implied less than $5 \times 10^{-3} \mathrm{M}_{\odot}$ of dust (somewhat dependent on the adopted grain material properties, very sensitive to the adopted clumpy distribution of the ejecta, and widely varying between $\mathrm{SNe}$ ), corresponding to an efficiency of $\simeq 10^{-3}-10^{-1}$ (Wooden et al. 1993, Sugarman et al. 2006, Ercolano et al. 2007, Meikle et al. 2007; see Barlow 2009 for a recent review). Young supernova remnants (SNR) provide another view of the dust formation efficiency of SNe and one that indicates much higher dust formation efficiencies. Spitzer studies of the young ( $\simeq 330 \mathrm{yr})$ supernova remnant, Cas A, revealed a warm dust mass of $0.025 \mathrm{M}_{\odot}$ in the volume processed by the reverse shock (Rho et al. 2008). A Herschel study adds to that $0.075 \mathrm{M}_{\odot}$ of cold dust mass interior to the reverse shock (Barlow et al. 2010). The total dust mass $\left(\simeq 0.1 \mathrm{M}_{\odot}\right)$ should be compared to the estimated total ejected mass of $2-4 \mathrm{M}_{\odot}$, much of this in the form of oxygen $\left(\simeq 2 \mathrm{M}_{\odot}\right)$ which will not condense (Willingale et al. 2002, Vink et al. 1996). In analogy to the well-studied type IIb SN 1993J, some $0.6 \mathrm{M}_{\odot}$ of condensible elements $(\mathrm{C}, \mathrm{Mg}, \mathrm{Si}, \mathrm{S} \& \mathrm{Fe})$ were ejected during the $\mathrm{SN}$ explosion of Cas A (Thielemann et al. 1996), corresponding to a very high dust formation efficiency of $\sim 0.2$. Likewise, Herschel observations of the extremely young SNR associated with SN 1987A - which has just entered the reverse shock phase as the ejecta slammed into the previous stellar wind remnant some 10 years after the explosion - reveal $\sim 0.5 \mathrm{M}_{\odot}$ 
Table 1. Inventory of dust in space

\begin{tabular}{lll}
\hline Oxide dust & Carbonaceous dust & Other \\
\hline amorphous silicates $(\mathrm{I}, \mathrm{C}, \mathrm{S})$ & PAHs (I,C,S) & silicon nitride $\mathrm{Si}_{3} \mathrm{~N}_{4}(\mathrm{~S})$ \\
crystalline forsterite, $\mathrm{Mg}_{2} \mathrm{SiO}_{4}(\mathrm{C}, \mathrm{S})$ & Fullerene, $\mathrm{C}_{60}(\mathrm{C}, \mathrm{I})$ & magnesium sulfide, $\mathrm{MgS}(\mathrm{C})$ \\
crystalline enstatite $\left(\mathrm{MgSiO}_{3}\right)(\mathrm{C}, \mathrm{S})$ & Amorphous Carbon (C,I,S) & Carbonate (C,I) \\
Silica, $\mathrm{SiO}_{2}(\mathrm{C})$ & Graphite (C,I,S) & Ice (C,I) \\
aluminum oxide, $\mathrm{Al}_{2} \mathrm{O}_{3}(\mathrm{C}, \mathrm{S})$ & Diamond (C,S) & \\
spinel, $\mathrm{MgAl}_{2} \mathrm{O}_{4}(\mathrm{C}, \mathrm{S})$ & silicon carbide, $\mathrm{SiC}(\mathrm{C}, \mathrm{I}$ ? ?,S $)$ & \\
titanium oxide, $\mathrm{TiO}_{2}(\mathrm{~S})$ & other carbides (C ?, S) & \\
hibonite, $\mathrm{CaAl} \mathrm{O}_{12} \mathrm{O}_{19}(\mathrm{~S})$ & & \\
Magnesium iron oxide, $\mathrm{Mg}_{0.1} \mathrm{Fe}_{0.9} \mathrm{O}(\mathrm{C})$ & & \\
\hline
\end{tabular}

Legend: I: Spectroscopic evidence for presence in interstellar dust. C: Spectroscopic evidence for presence in circumstellar dust. S: Present as stardust in meteoritic or cometary material (For a a discussion, see Tielens 2001, Zinner 2003).

of cold dust $†$ (Matsuura et al. 2011); many orders of magnitude larger than estimated from observations during the dust condensation period of $\sim 500-1000$ days (Wooden et al. 1993). Given the bewildering zoo of SNe types and the uncertainties and conflicting observational results on dust formation in these environments, the contribution of SNe to the dust budget can presently only be guessed at but this anecdotal evidence suggests that it is high. Figure 2 assumes that all of the condensibles form dust and type II SNe are then more important than AGB stars for the dust budget.

Figure 2 also includes a contribution of dust formed in the inner regions of protoplanetary disks and entrained and ejected by the protostellar wind. This estimate is also at the high end as it is based upon the assumption that $1 / 3$ of the accreting mass is ejected as a wind (Shu \& Shang 1997) and that all condensibles form dust. Other estimates of protostellar wind characteristics typically result in a $\sim 3$ times smaller wind mass loss rate (Hartmann 1995). It should also be kept in mind that, for the dust budget, protostellar winds are only a pseudo source of dust. The wind likely originates from the inner region where all preexisting dust has sublimated and recondensed. The net addition of dust may then actually be negative (carbon will not condense as dust in these environments). Certainly, in terms of the stardust budget, protostars present a sink.

In summary, while the details are heatedly debated, it is clear that many different sources contribute to the dust budget of the ISM of galaxies.

\section{Composition of stardust}

The composition of interstellar dust can be studied through IR spectroscopy and through the analysis of genuine stardust grains retrieved from meteorites (Tielens 2001, Zinner 2003). These records show that the composition of circumstellar dust is rich and varied (Table 1) with silicates and other oxides, various forms of pure carbon-based materials and carbides, and a variety of other materials. In the astronomical record, much of this heterogeneity refers to circumstellar dust either in late type stars returning much of their material back to the ISM or in (the disks of) young stellar objects such as T Tauri stars and Herbig AeBe stars. Interstellar dust mainly consists of amorphous silicates and amorphous carbon. Limits on other materials are at the percent level (Kemper

$\dagger$ As this was observed after the SN ejecta reached the blue supergiant ejecta and the reverse shock has started to process the SN material, contamination by line emission may have affected this estimate. 
et al. 2004). In addition, a substantial fraction $(\sim 0.05)$ of the elemental carbon in the Universe is locked up in PAHs and a much smaller fraction $\left(10^{-5}-10^{-4}\right)$ in the fullerene, $\mathrm{C}_{60}$. (Tielens 2005, Sellgren 2010, Berne \& Tielens 2011).

\section{Processing of dust in the interstellar medium}

\subsection{Shock destruction}

Interstellar shocks destroy interstellar dust (Jones et al. 1994, Jones et al. 1996) mainly through sputtering by impinging energetic ions. Supernova explosions drive strong shock waves into the surrounding interstellar medium. As the supernova remnant expands, the shock velocity drops. Initially, this expansion is adiabatic (the Sedov-Taylor phase) because very hot gas cools slowly, but when the shock velocity drops below $\simeq 250 \mathrm{~km} / \mathrm{s}$, cooling becomes important (the radiative phase). Eventually, the remnant will merge with the ISM. Near threshold, the sputtering yield of materials rises steeply reaching a broad maximum of $\sim 10^{-2}$ for $\mathrm{H}$ atoms impacting with $\sim 1 \mathrm{keV}$. Because a much larger volume of the ISM is processed by low velocity shocks than by fast shocks, most of the destruction is done by those radiative shocks where impacting $\mathrm{H}$-atoms are near the threshold of the sputtering yield (e.g., $v_{s} \simeq 10^{2} \mathrm{~km} / \mathrm{s}$ ). Because, for a given SNR pressure, the shock velocity in a phase scales with $\rho^{1 / 2}$, most of the destruction will occur by $100 \mathrm{~km} / \mathrm{s}$ shocks in the tenuous intercloud medium (rather than by shocks propagating in the diffuse cloud phase). In the plane of the Milky Way, such shocks occur typically once every sojourn of the grain into the warm intercloud medium and scrapes off some $30 \AA$ from a grain surface. The destruction timescale is then set by the number of shocks required to erode a grain "fully" which depends on the interaction of supernovae with the ISM. Estimated timescales are $\simeq 500 \mathrm{Myr}$ and are not very sensitive to the details of the model for the ISM (e.g., 2-phase, 3-phase; Jones et al. 1994).

\subsection{Interstellar depletions}

This timescale for dust destruction, $\tau_{\text {des }}$, is much shorter than the timescale for replenishment of dust material by old, dying stars $\left(\tau_{i n j} \simeq 3 \mathrm{Byr}\right)$. Hence, in a steady state between dust injection by stars and destruction by shocks in the ISM, the expected depletion of condensible elements is $\delta / \delta_{o}=\left(1+\tau_{i n j} / \tau_{\text {des }}\right)^{-1} \simeq 0.15$ where $\delta_{o}$ is the fraction of the element injected in the form of dust by stars. This is much less than the observed depletion of such dust-forming elements as Mg, Si, and Fe (0.9-0.99; Savage \& Sembach 1996). Indeed, in such a model, the high observed depletions would require/imply a destruction timescale $\tau_{d e s}>>\tau_{i n j}$ and that is incompatable with sputtering studies of materials and the "violent" nature of the ISM. In fact, some $10 \%$ of the condensible elements are injected into the ISM by stars (OB \& WR stars) which do not form dust at all and hence such a steady state model can never explain depletions in excess of 0.9 (e.g., depletions of elements such as Fe, Ti, Ca; Jura 1987).

It is clear that dust has to regrow very quickly in the ISM itself and there is direct observational evidence for this. Observations have revealed a large and systematic difference in the elemental depletions in the different phases of the interstellar medium (Fig. 5.2; Savage \& Sembach 1996, Cartledge et al. 2006) and these variations can be used to get an "observational" handle on the rate at which dust is destroyed in the intercloud medium and reformed through accretion in the cloud phase. The simple model for interstellar depletions, outlined above, can be extended to include accretion of elements in the cloud phase of the ISM; so, there is now a balance between dust injection by stars, dust destruction in the Warm Intercloud Medium, and accretion in the (diffuse) cloud phase of the ISM (Tielens 1998). The observed large depletion variations demonstrate 


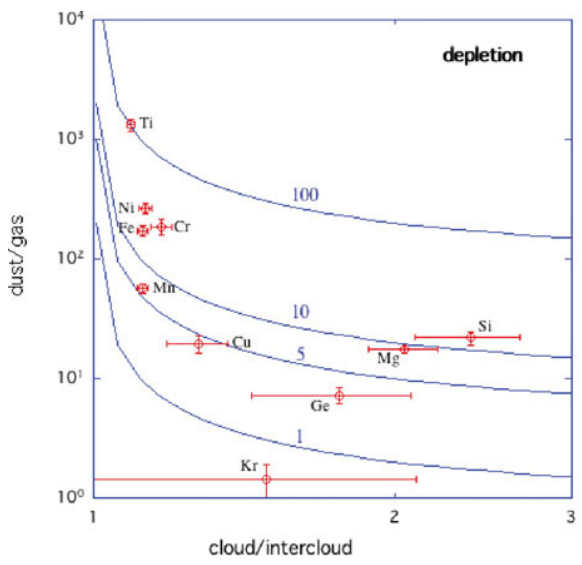

Figure 3. Observed depletions of elements in the ISM (Savage \& Sembach 1996, Jenkins 2009). The $y$-axis is the ratio of the abundance of an element in the dust phase to its abundance in the gas phase, both measured in diffuse clouds. The $\mathrm{x}$-axis is the ratio of the depletion of these elements in the diffuse cloud medium to that in the intercloud medium. The solid lines are the results of a simple model balancing destruction in the intercloud medium with accretion in the cloud medium (Tielens 1998). The labels indicate the adopted values for the accretion rate relative to the cloud-to-intercloud mixing ratio. As these observations demonstrate, the rates for destruction in the intercloud phase, accretion in the cloud phase, and mixing between these phases have to be within a factor of a few of each other and destruction and accretion are thus very rapid compared to the injection rate of dust by stars into the ISM.

rather directly that the processes involved - shock destruction and accretion - operate on a timescale similar to the timescale at which material is mixed from the cloud to the intercloud medium and back (Fig. 5.2). This mixing timescale is much less $(\simeq 30$ million years) than the timescale at which new dust is injected into the ISM. Thus, specifically, some $10 \%$ of the iron, $15 \%$ of the magnesium, and some $30 \%$ of the silicon is returned to the gas phase upon each sojourn into the intercloud medium and then rapidly reaccreted once the material is cycled back to the (diffuse) cloud phase. Again, as for the material injected into the ISM by non-dust-producing stellar sources, this points towards the importance of the formation of a thin, outer layer in the ISM. This thin coating is readily sputtered in the warm intercloud medium and in that way protects underlying (stardust) grains against the destructive effects of shocks.

\subsection{Noble gasses in stardust grains}

Genuine stardust grains have been isolated from carbonaceous meteorites. Analysis of these stardust grains has revealed an isotopic composition which is distinctly non-Solar and derives directly from the stellar birthsites of these dust grains (Anders \& Zinner 1993). Apparently, these dust grains formed in stellar ejecta - enriched by the nucleosynthetic products of processes taking place in the deep interiors of these stars and then mixed to the surface - were injected into the ISM, processed by shocks and other energetic events, became part of a region of star formation that collapsed to form the Solar system, saw the hot gases swirl in the Solar nebula, experienced possibly the shocks and lightning processes rampant in this environment, were incorporated into the planetary body from which the meteorite was derived, survived the violent break-up of this body, crashed on Earth and then was taken apart in the laboratory; and through all this arduous and torturous history, these stardust grains never equilibrated fully with the gas and managed to preserve their stellar heritage. Detailed studies of these stardust grains have opened a new window on the dusty universe, providing new insights in the composition 

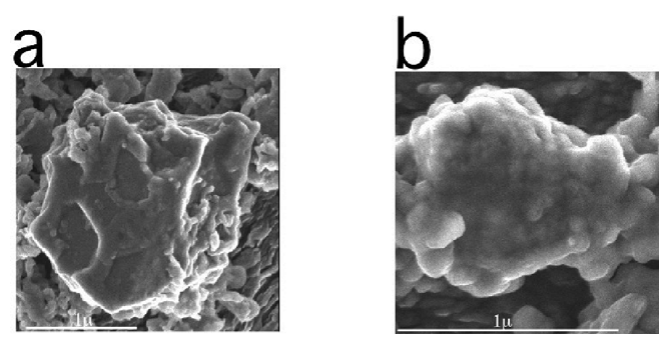

Figure 4. Field emission scanning electron microscope images of pristine presolar SiC grains from the Murchison meteorite (Bernatowicz et al. 2003): (a) exhibiting primary growth crystal faces and polygonal depressions; (b) coated with an apparently amorphous, possibly organic phase. Scale bars are $1 \mu \mathrm{m}$.

of dust and the processes that play a role in their formation (Bernatowicz et al. 2003). In addition, recent studies have started to explore the impact of interstellar processes on these grains (Henkel et al. 2010, King et al. 2010). Here, I will focus on the latter aspect. SiC stardust contains appreciable amounts of noble gasses and analysis of the isotopic composition of these noble gasses reveals the presence of two distinct components: The G-component characteristic for the isotopic composition of AGB stars (e.g., s-process) and the N-component with a solar system/ISM isotopic composition (Lewis et al. 1994). The abundances of each of these two components show fractionation with the lighter elements less abundant than expected from the abundance of Xenon. This fractionation is size dependent with smaller grains showing more severe fractionations. Noble gasses trapped in $\mathrm{SiC}$ grains represent the effects of ion implantation and the implantation depth is very energy dependent. For a constant grain-gas relative velocity, heavier species are more energetic and therefore implant deeper. The G-component is then thought to result from implantation of AGB-material during the PN nebula phase when radiation pressure rapidly accelerates grains to velocities of $\simeq 250 \mathrm{~km} / \mathrm{s}$ relative to the gas. The N-component is the result of implantation during $\sim 100 \mathrm{~km} / \mathrm{s}$ shock processing in the ISM (Guillard et al. 2011). The implantation of the N-component is accompanied by sputtering of the surface layer and it is this sputtering that preferentially removes the shallowier implanted, lighter noble gasses and leads to fractionation. The results for a simple model are compared to the measurements in Figure 5. Thus, the measured fractionation of the noble gases preserved in $\mathrm{SiC}$ stardust grains provide rather direct evidence for the shock processing of dust in the interstellar medium. Additional evidence for the importance of ion implantation is provided by depth-dependent studies of the concentration of trace elements in a few individual stardust grains. These reveal a concentration towards the surface as expected for ion-implantation at velocities of $\sim 500 \mathrm{~km} / \mathrm{s}$ (Henkel et al. 2010, King et al. 2010).

\section{Dust formation in the ISM}

This review emphasizes the importance of dust growth in the ISM. For some elements (the heavily depleted ones: $\mathrm{Mg}, \mathrm{Si}, \mathrm{Fe}, \mathrm{Ca}, \mathrm{Al}, \mathrm{Ti}, \ldots$ ), this is an efficient process. But other elements are hardly affected. Sodium and potasium are obvious examples of elements that are never highly depleted. Oxygen seems to be involved to some extent but sulfur not. Most telling perhaps, carbon does not seem to show a difference in depletion between the warm intercloud medium and the diffuse cloud phase of the ISM and oxide rather than carbonaceous mantles seem indicated. From shock models, the average binding energy of atoms in this mantle is $\simeq 2 \mathrm{eV}$ (Tielens 1998) and they are quite refractory. Past models 

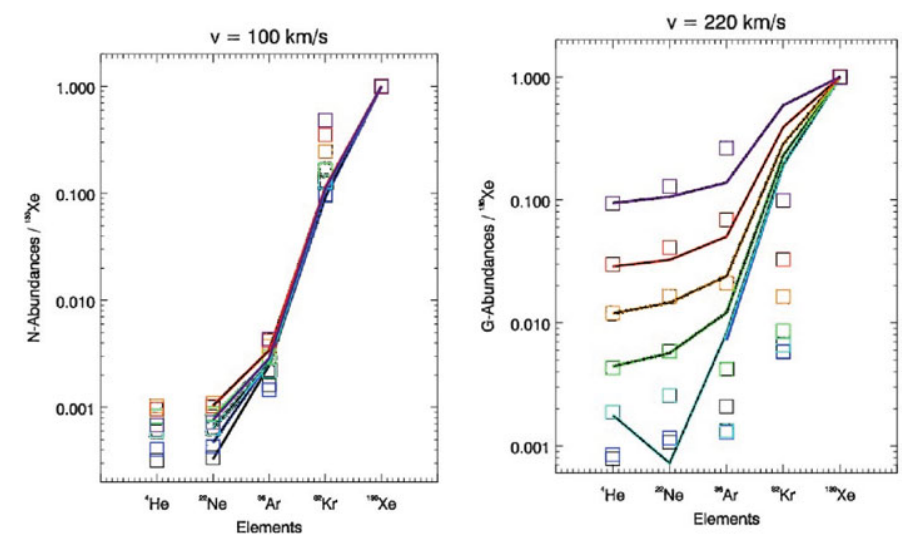

Figure 5. Comparison between measured noble gas abundances (symbols) in $\mathrm{SiC}$ grains extracted from the Murchison meteorite and model predictions (lines) for the N- (Left) and G(Right) components. Elemental abundances have been normalized to ${ }^{130} \mathrm{Xe}$ and to Solar abundances for $\mathrm{N}$ or AGB abundances for $\mathrm{G}$. Each line refers to a different size fraction (top to bottom: big to small). Error bars are smaller than the symbols (Guillard et al. 2011).

have invariably focused on carbonaceous mantles formed by energetic processing (e.g., UV photolysis or ion bombardment) of simple molecular ice mixtures in dense molecular clouds. However, while organic "goop" is readily formed this way in the laboratory, there is no observational evidence for the importance of this process in the ISM.

The chemistry of mantle formation seem to be very different. Likely, this is because this oxide mantle is predominantly formed in the diffuse ISM. A number of effects may then play a role (Tielens \& Allamandola 1987). The preponderance of atomic hydrogen may favor the formation of simple hydrides and the more volatile ones $\left(\mathrm{H}_{2} \mathrm{O}, \mathrm{CH}_{4}, \ldots\right)$ are readily photodesorbed. Other atoms may preferentially form coordination complexes or salts, which are not so susceptible to photo-erosion. Moreover, the high UV photon flux may promote chemical interlinking of the growing surface layer and the underlying material. Of course, the high degree of ionization may also have some influence on the accretion/sticking rate; particularly for elements with ionization potentials below the grain work function $(\mathrm{Na}, \mathrm{K})$. At this point, these are mere speculations and a systematic study of the chemical routes and kinetics of grain surface chemistry is called for. The large body of data on interstellar elemental depletions may well provide a good guide for such endeavors.

\section{Future}

A good understanding of the characteristics of interstellar dust is a prerequisite for the interpretation of the spectral energy distribution of galaxies. We have a global understanding of the processes involved and some of these processes have been studied in great detail. It is clear that interstellar dust is very diverse, has many sources, and is heavily processed in the ISM. Amorphous silicates and some form of aromatic carbon dust are key components of interstellar dust. The properties of the dust will reflect local conditions, including the relative importance of different stardust sources, the evolution of SNR and the rate at which they process the ISM, and the detailed chemistry involved in mantle formation in the ISM.

The characteristics of dust at high $\mathrm{z}$ is of particular interest. At present, this is highly uncertain and the role of dust in the early evolution of the Universe can only be guessed at. 
From studies in the (relatively) local Universe, it is obvious that the properties of dust are very dependent on its environment. The UV extinction curves in the Magellanic Clouds are quite different from those in the local solar neighborhood (Cartledge et al. 2005), either because of the large fraction of C-rich AGB stars or because SNe can process the ISM more readily in these dwarf galaxies. Likewise, dust in the extreme environments of ULIRG and quasar nuclei is very different from that in the Milky Way (Marwick Kemper et al. 2005, Spoon et al. 2006) either because it is formed in massive star or quasar outflows or because stardust is heavily processed in these energetic environments. Much has been made in recent studies on whether (the high mass end of) the low mass stars can evolve rapidly enough to produce the observed dust at high redshifts in their AGB phase or whether SN are the dominant producers of this dust. It is important to realize that this may actually be very moot point. In the high star formation environment of the early Universe, SNR may rapidly process any stardust and the dust we see could largely be produced by the chemical accretion processes discussed above for the Milky Way.

Despite our general ignorance in all matters dusty, the future looks bright. Recent studies of the stardust budget have focuses on the Magellanic clouds where the SAGE Spitzer Legacy programs have been very successful (Sargent et al. 2010, Mikako et al. 2010). With the launch of Gaia, accurate distances of stars in the Milky Way can be determined, removing the largest uncertainty in studies of the dust mass budget of the Milky Way. With SOFIA operational, well-determined mass loss rates for various classes of AGB stars and supergiants are then in sight for our own galaxy. The James Webb Space Telescope (JWST) can extend this to galaxies in the local group. Most importantly, JWST will be able to survey IR emission of all SNe \& LBV within $50 \mathrm{Mpc}$ and determine masses and composition of dust formed in the ejecta. This volume is large enough that all denizens of the SN-zoo can be reasonably expected to be probed. JWST can also systematically probe characteristics of dust in regions of extreme star formation. The long wavelength capabilities of Herschel, SOFIA and SPICA will be essential to probe cold dust characteristics in young SNR, while warm dust (in the reverse shock) can be studied by JWST and SPICA. Linking these to the characteristics of the progenitor and the conditions (eg., elemental composition) of the dusty knots may then shed much light on the interaction of SN with the dust they produce. The contours of the dusty Universe, observed through a looking glass darkly in this review, may then well focus in full glory.

\section{References}

Anders, E. \& Zinner, E. 1993, Meteoritics, 28, 490

Barlow, M. J., Krause, O., Swinyard, B. M., et al. 2010, A\&A, 518, L138

Barlow, M. J. 2009, Astrophysics in the Next Decade, (Berlin: Springer Verlag), 247

Bernatowicz, T. J. et al. 2003, Geochim. Cosmochim. Acta, 67, 4679

Berné, O. \& Tielens, A. G. G. M. 2011, PNAS, in press

Bertoldi, F., Carilli, C. L., Cox, P., et al. 2003, A\&\&A, 406, L55

Cartledge, S. I. B., Clayton, G. C., Gordon, K. D., et al. 2005, ApJ, 630, 355

Cartledge, S. I. B., Lauroesch, J. T., Meyer, D. M., \& Sofia, U. J. 2006, ApJ, 641, 327

Cherchneff, I. \& Dwek, E. 2010, ApJ, 713, 1

Cherchneff, I. 2000, The Carbon Star Phenomenon, 177, 331

Ercolano, B., Barlow, M. J., \& Sugerman, B. E. K. 2007, MNRAS, 375, 753

Guillard, P., Jones, A. P., \& Tielens, A. G. G. M. 2011, A\& $\mathcal{G}$, submitted

Hartmann, L. 1995, Revista Mexicana de Astronomia y Astrofisica Conference Series, 1, 285

Henkel, T., King, A., \& Lyon, I. 2007, Lunar and Planetary Institute Science Conference Abstracts, 38, 2351 
Jenkins, E. B. 2009, ApJ, 700, 1299

Jones A. P., Tielens, A. G. G. M., Hollenbach, D. J., \& McKee, C. F. 1994, ApJ, 433, 797

Jones, A. P., Tielens, A. G. G. M., \& Hollenbach, D. J. 1996, ApJ, 469, 740

Jura, M. 1987, in "Interstellar Processes", ASSL, 134, p3

Kemper, F., Vriend, W. J., \& Tielens, A. G. G. M. 2004, ApJ, 609, 826

King, A. et al. 2010, Lunar and Planetary Institute Science Conference Abstracts, 41, 1976

Lewis, R. S., Amari, S., \& Anders, E. 1994, GeCoA, 58, 471

Lucy, L. B. et al. 1989, in "structure and dynamics of the interstellar medium", eds. G. TenorioTagle, M. Moles, J. Melnick (Berlin: Springer Verlag), p164

Markwick-Kemper, F., Gallagher, S. C., Hines, D. C., \& Bouwman, J. 2007, ApJ, 668, L107

Matsuura, M., et al. 2011, Science, 333, 6047

Meikle, W. P. S., Mattila, S., Pastorello, A., et al. 2007, ApJ, 665, 608

Matsuura, M., Barlow, M. J., Zijlstra, A. A., et al. 2009, MNRAS, 396, 918

Rho, J., Reach, W. T., Tappe, A., et al. 2009, ApJ, 700, 579

Rho, J., Kozasa, T., Reach, W. T., et al. 2008, ApJ, 673, 271

Sargent, B. A., Srinivasan, S., Meixner, M., et al. 2010, ApJ, 716,

Savage, B. D. \& Sembach, K. R. 1996, Ann Rev Astron Astrophys, 34, 279

Sellgren, K., et al. 2010, ApJ, 722, L54

Shu, F. H. \& Shang, H. 1997, Herbig-Haro Flows and the Birth of Stars, 182, 225

Spoon, H. W. W., Tielens, A. G. G. M., Armus, L., et al. 2006, ApJ, 638, 759

Sugerman, B. E. K. et al. 2006, Science, 313, 196

Thielemann F. K., Nomoto, K., \& Hashimoto, M-A. 1996, ApJ, 460, 408

Tielens, A. G. G. M. \& Allamandola, L. J. 1987, in "Interstellar Processes", ASSL, 134, p333

Tielens, A. G. G. M. 1998, ApJ 499, 267

Tielens, A. G. G. M. 2001, in Tetons 4: Galactic Structure, Stars and the Interstellar Medium, eds. C. E. Woodward, M. D. Bicay, and J. M. Shull (San Francisco, ASP) 231, 92

Tielens, A. G. G. M. 2005, Physics and Chemistry of the Interstellar Medium, (Cambridge: University of Cambridge Press)

Vink, J. et al. 1996, A\& A, 307, L41

Willingale, R., et al. 2002, A\&A, 381, 1039

Wooden, D., et al. 1993, ApJS, 88, 477

Zinner, E. 1998, Ann. Rev. Earth Planet. Sci., 26, 147

Zinner, E. K. 2003, In Treatise on Geochemistry Vol 1, ed. K. K. Turekian, H. D. Holland, A. M. Davis), (Amsterdam: Elsevier), p17

\section{Discussion}

Henkel: Traditionally, the production of the rare nitrogen isotope $15 \mathrm{~N}$ was tentatively attributed to novae. In your plot of carbon and nitrogen isotope ratios from individual dust grains, however, grains related to novae show particularly large $14 \mathrm{~N} / 15 \mathrm{~N}$ ratios. Does this imply that $15 \mathrm{~N}$ production by novae can be discarded?

Tielens: Nucleosynthesis is beyond my expertise. However, the indicated nova grain in the stardust isotope data are characterized by low N14/N15 and low C12/C13 ratios. They also show excess Si30, and high 26Al/27Al. This indicates explosive hydrogen burning and a likely origin in nova.

TuFfs: Do you think it is feasible to condense hydrogen onto grain cores - can you get cold enough?

TiELEns: The key point is indeed the dust temperature. The sublimation temperature of solid $\mathrm{H} 2$ is $\sim 4 \mathrm{~K}$, which is only slightly higher than the background temperature of $2.7 \mathrm{~K}$. So, dust really does not get that cold, observationally. Dust in the diffuse ISM is at $\sim 16-20 \mathrm{~K}$. Dust in dense clouds may get as cold as $6 \mathrm{~K}$. 